\title{
QUASI-PERIODIC DYNAMICS OF DESINGULARIZED VORTEX MODELS
}

\author{
Chjan C. LIM* \\ Department of Mathematics, University of Michigan, Ann Arbor, MI 48109, USA
}

\begin{abstract}
Sufficient conditions for the existence of quasi-periodic solutions of two different desingularized vortex models for 2-dimensional Euler flows are derived. One of these models is the vortex blob model for the evolution of a periodic vortex sheet and the other is a second order elliptic moment model (DEMM) for the evolution of widely separated vortex regions. The method involves the identification of the well-known point vortex Hamiltonian term in both models. A transformation to new canonical variables (the JL-coordinates) and the definition of special open sets in phase space (the cone sets) puts the Hamiltonians considered into nearly integrable form. KAM-theory is used to prove the desired results for arbitrary degrees of freedom and almost arbitrary circulations in these models. A rigorous validification of the DEMM assumption is nbtained. In view of the lack of a rigorous theory for vortex sheet roll-up past the critical time, the dynamical system approach presented here provides an alternative method for studying the macroscopic structures formed in the post-critical period.
\end{abstract}

\section{Introduction}

Recently, there is a lot of research activity on vortex methods for the two-dimensional Euler equation of ideal fluids. In particular, Krasny [1,2] has computed the evolution of a periodic vortex sheet by two methods: a point vortex lattice method and a desingularized vortex blob method. The first method gives good results before singularity formation but the second method can be used beyond $t_{c}$ to demonstrate vortex sheet roll-up. Convergence before $t_{c}$ of the nethods for vortex sheet has been shown by Caflisch and Lowengrub [3] in a recent paper. There is currently no rigorous proof that the vortex blob method converge beyond singularity formation at time $t_{c}$. However numerical evidence for its convergence is available and as conjectured by Pullin [4], the vortex sheet rolls up into a double branched spiral (cf. Saffman and Baker [5] for other references). In view of the absence of a complete theory, a dynamical system approach for studying the solutions of these vortex models is reasonable. A statistical mechanics for these models will perhaps demonstrate (along the lines of Montgomery et al. [6, 7]) that "most probable states" are non-uniform distributions of vortex blobs. Here we give some rigorous results on the existence of quasi-periodic solutions for the vortex blob method. Complete proofs for a variety of point vortex models are reported in $\operatorname{Lim}[8,9]$ and numerics in $\operatorname{Lim}$ and Sirovich [10]. It should be noted (for motivation of this approach), that the "large sets" of quasi-periodic solutions correspond to clustered states of vortex blobs.

The second vortex model for two-dimensional Euler flow that we discuss here is the Desingularized Elliptic Moment Model (DEMM) of Melander, Zabusky and Styczek [11]. They perform a perturbation analysis based on the assumption that the finite uniform-vorticity regions are remote from each other and nearly circular. A consistent truncation then yields an arbitrary $N$ th order model for the moments of the

*Address for academic year 1988-1989: Institute of Mathematics and Applications, University of Minnesota. Minneapolis, USA. 
vorticity regions. Yiv focus on the second order model where each region is assumed elliptical (DEMM). The DEMl. can be used to follow the evolution of vorticity regions anywhere in an unbounded fluid. Furthermore this model conserves local area, global centroid, total angular impulse and global excess energy. Of special significance is the fact that the model can be written in canonical Hamiltonian form. In this note we prove the existence of a "large set" of quasi-periodic solutions for DEMM. As a corollary, we give a rigorous validification of the DEMM for a large set of initial data. Its validity has been confirmed numerically [11].

\section{The method}

We use a general method developed in $\operatorname{Lim}[9,12]$. It consists of the following steps:

(i) Under additional assumptions, we first show that the Hamiltonians for the above vortex models can be written as a small perturbation of the $N$-point vortex Hamiltonian (pius a completely integrable term for DEMM),

$$
H_{\mathrm{p}}=\frac{-1}{4 \pi} \sum_{1}^{N} \Gamma_{j} \Gamma_{k} \log \left|z_{j}-z_{k}\right|^{2}
$$

(ii) Next, we define new canonical variables, the JL-coordinates for vortex dynamics (see fig. 1):

$$
\begin{aligned}
& \rho_{1}=\left[\frac{\Gamma_{1} \Gamma_{2}}{\Gamma_{1}+\Gamma_{2}}\right]^{1 / 2} \rho_{1}^{\prime}, \\
& \rho_{2}=\left[\frac{\left(\Gamma_{1}+\Gamma_{2}\right) \Gamma_{3}}{\Gamma_{1}+\Gamma_{2}+\Gamma_{3}}\right]^{1 / 2} \rho_{2}^{\prime}, \\
& \rho_{N-1}=\left[\frac{\left(\sum_{j=1}^{N-1} \Gamma_{j}\right) \Gamma_{N}}{\sum_{j=1}^{N} \Gamma_{j}}\right]^{1 / 2} \rho_{N-1}^{\prime},
\end{aligned}
$$$$
\rho_{1}^{\prime}=z_{2}-z_{1}
$$

$$
\begin{aligned}
& \rho_{2}^{\prime}=z_{3}-\frac{\Gamma_{1} z_{1}+\Gamma_{2} z_{2}}{\Gamma_{1}+\Gamma_{2}}, \\
& \rho_{\mathrm{N}-1}^{\prime}=z_{N}-\frac{\sum_{1}^{N-1} \Gamma_{j} z_{j}}{\sum_{1}^{N-1} \Gamma_{j}} .
\end{aligned}
$$

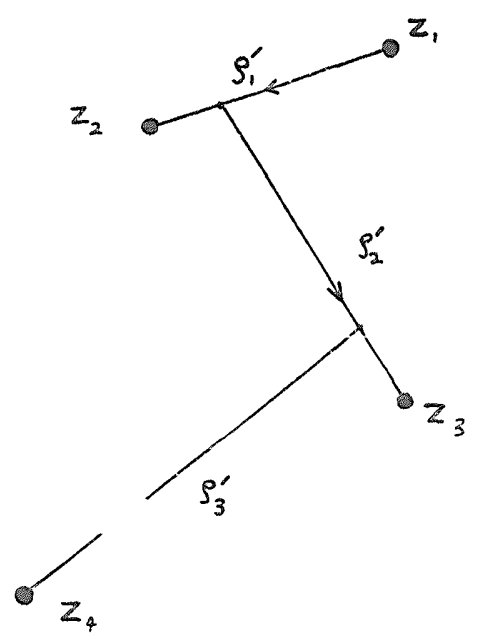

Fig. 1. The JL-coordinates. The $N=4$ case when all the circulations have the same sign is illustrated. 
(iii) Based on an ordering of the JL-coordinates $\rho_{k}$ by modulus $\left|\rho_{k}\right|$, we construct cone sets in phase space in which the $N$-point vortex Hamiltonian (2.1) can be written as a small perturbation of the Hamiltonian for $(N-1)$ decoupled oscillators.

(iv) In the last step, an application of KAM-theory $[13,14]$ gives sufficient conditions on the circulations $\Gamma_{k}$ for the existence of quasi-periodic solutions.

In the case of vorticity of one sign, the JL-coordinates reduces to the Jacobi coordinates often encountered in celestial mechanics papers. Referring to the $N=4$ case depicted in fig. 1 , one can easily conclude that the canonical transformation (2.2) gives a one-to-one mapping from the $M=N(N-1) / 2$ edges of the complete graph $G$ for $N$ vertices $\left\{z_{i}\right\}_{i=1}^{N}$ onto the paths of the corresponding directed tree graph $G^{\prime}$. For two species of vorticity, the tree graphs $G^{\prime}$ are much more complicated and we refer the reader to $\operatorname{Lim}[9]$ for the complete picture. The above JL-coordinates belong to a large family of canonical transformations for $N$-body problems recently discovered by this author; their relation to a class of spanning tree graphs is discussed in Lim [17].

\section{3. $N$-point vortex Hamiltonian}

In this section, we briefly review steps (ii) and (iii) in the outline above. The details can be found in $\operatorname{Lim}$ [9]. This part of the method represents the common core of the analyses for the vortex blob model as well as the DEMM. Step (i) of the method (when completed in sections 4 and 5 for the two models respectively), provides rigorous justification that the $N$-point vortex Hamiltonians (2.1) is fundamental to the dynamics of a number of vortex modess, both singular and desingularized ones.

After transformation to the JL-coordinates, the Hamiltonian (2.1) is reduced to $(N-1)$ degrees of freedom:

$$
\begin{gathered}
H_{\mathrm{p}}=\frac{1}{4 \pi} \sum_{j=1}^{M} K_{j} \log \left|f_{j}\left(\rho_{1}, \ldots, \rho_{j}\right)\right|^{2}, \\
K_{j}=\Gamma_{p} \Gamma_{q}, \quad M=N(N-1) / 2,
\end{gathered}
$$

where $f_{j}$ are linear functions in the complex variables $\rho_{k}, k \leq j$. A typical term is

$$
\begin{aligned}
z_{3}-z_{1} & =f_{2}\left(\rho_{1}, \rho_{2}\right) \\
& =\left[\frac{\Gamma_{1}+\Gamma_{2}+\Gamma_{3}}{\left(\Gamma_{1}+\Gamma_{2}\right)\left(\Gamma_{3}\right)}\right]^{1 / 2} \rho_{2}+\left[\frac{\Gamma_{2}}{\left(\Gamma_{1}\right)\left(\Gamma_{1}+\Gamma_{2}\right)}\right]^{1 / 2} \rho_{1}
\end{aligned}
$$

In special open sets called cone sets given by

$$
M_{\delta}=\left\{\rho=\left(\rho_{1}, \ldots, \rho_{N-1}\right)|0<| \rho_{1}|<\Delta| \rho_{2}\left|<\cdots<\Delta^{N-2}\right| \rho_{N-1} \mid\right\}, \Delta \ll 1,
$$


the Hamiltonian (3.1) can be written as a small perturbation in the following way:

$$
\begin{aligned}
& H_{\mathrm{p}}\left(\rho_{1}, \ldots, \rho_{N-1}\right)=H_{0}(\rho)+H_{1}(\rho) \\
& H_{0}(\rho)=-\frac{1}{4 \pi} \sum_{j=1}^{N-1} T_{j} \log \left|\rho_{j}\right|^{2} \\
& T_{j}=\Gamma_{j+1} \sum_{k=1}^{j} \Gamma_{k}, \quad j=1, \ldots, N-1, \\
& H_{1}(\rho)=-\frac{1}{4 \pi} \sum_{j=1}^{M} K_{j} \log |(1+O(\Delta))|^{2}
\end{aligned}
$$

The leading term $H_{0}$ is a decoupled Hamiltonian which implies that it is completely integrable. All solutions have the form of products

$$
T^{N-1}=S^{1} \times S^{1} \times \cdots S^{1} \quad(N-1) \text { times. }
$$

This simple Hamiltonian for decoupled oscillators represents the key to our analyses. In so far as the $N$-point vortex Hamiltonian (2.1) can be found in many vortex models, the identification of $H_{0}(3.4 \mathrm{~b})$ (via convergent expansions) is the motivation as well as the main tool for a dynamical study of a variety of problems.

\section{The vortex blob Hamiltonian for periodic vortex sheet}

The desingularized Birkhoff equations for a periodic vortex sheet are [2]

$$
\begin{aligned}
& \frac{\partial x}{\partial t}=-\frac{1}{2} \int_{0}^{1} \frac{\sinh 2 \pi\left(y-y^{\prime}\right) \mathrm{d} \Gamma^{\prime}}{\cosh 2 \pi\left(y-y^{\prime}\right)-\cos 2 \pi\left(x-x^{\prime}\right)+\delta^{2}}, \\
& \frac{\partial y}{\partial t}=\frac{1}{2} \int_{0}^{1} \frac{\sinh 2 \pi\left(x-x^{\prime}\right) \mathrm{d} \Gamma^{\prime}}{\cosh 2 \pi\left(y-y^{\prime}\right)-\cos 2 \pi\left(x-x^{\prime}\right)+\delta^{2}},
\end{aligned}
$$

where $x=x(\Gamma, t), y=y(\Gamma, t)$ and $x^{\prime}=x\left(\Gamma^{\prime}, t\right), y^{\prime}=y\left(\Gamma^{\prime}, t\right) . \Gamma$ is a Lagrangian parameter which measures the total circulation between a fixed material point and an arbitrary material point along the curve (Birkhof [15]); $\imath$ is time and $\delta>0$ is a measure of the desingularization or "size" of the vortex blob in the following spatial discretization. The usual discretization approximates the curve $(x,(\Gamma, t), y,(\Gamma, t), 0 \leq \Gamma$ $\leq 1$ by $N$ points $\left(x_{j}(t), y_{j}(t)\right)$ on a uniform $\Gamma$-mesh, yielding

$$
\begin{aligned}
& \frac{\mathrm{d} x_{j}}{\mathrm{~d} t}=-\frac{1}{2 N} \sum_{k=1}^{N} \frac{\sinh 2 \pi\left(y_{j}-y_{k}\right)}{\cosh 2 \pi\left(y_{i}-y_{k}\right)-\cos 2 \pi\left(x_{j}-x_{k}\right)+\delta^{2}}, \\
& \frac{\mathrm{d} y_{j}}{\mathrm{~d} i}=\frac{1}{2 N} \sum_{k=1}^{N} \frac{\sinh 2 \pi\left(y_{j}-y_{k}\right)}{\cosh 2 \pi\left(y_{i}-y_{k}\right)-\cos 2 \pi\left(x_{j}-x_{k}\right)+\delta^{2}} .
\end{aligned}
$$


We will consider a more general Hamiltonian which takes into account arbitrary circulations $\Gamma_{k}$. namely,

$$
H_{\mathrm{B}}=-\frac{1}{4 \pi} \sum_{j=1}^{N} \sum_{k>j} \Gamma_{k} \Gamma_{j} \log \left(\cosh 2 \pi\left(y_{j}-y_{k}\right)-\cos 2 \pi\left(x_{j}-x_{k}\right)+\delta^{2}\right)
$$

For constant circulations, $\Gamma_{k}=1 / N$, consistent with the above discretization procedure, $H_{\mathrm{B}}$ reduces to the Hamiltonian for the system (4.2) with conjugate variables

$$
\left(\frac{x_{j}}{\sqrt{N}}, \frac{y_{j}}{\sqrt{N}}\right), \quad j=1, \ldots, N
$$

Borgers [16] recently proved the convergence for solutions of (4.2) as $N \rightarrow \infty$. A stronger result was proved independently by Caflisch and Lowengrub [3]. Their result is uniform in $\delta$, allowing them to recever the convergence result for the point vortex lattice method [1] as $\delta$ tends to zero. They also proved the global existence of analytic solutions to (4.1).

We are now ready to carry out step (i) in our method. Expanding cosh and cos in powers of their respective arguments, we obtain

$$
\begin{aligned}
& H_{\mathrm{B}}=-\frac{1}{4 \pi}\left\{\sum_{j=1}^{N} \sum_{k>j} \Gamma_{k} \Gamma_{j} \log R_{j k}^{2}+\sum_{j=1}^{N} \sum_{k>j} \log \left(1+\frac{\delta^{2}}{2 \pi^{2} R_{j k}^{2}}+\mathcal{O}\left(R_{j k}^{3}\right)\right)\right\}, \\
& R_{j k}^{2} \equiv\left(x_{j}-x_{k}\right)^{2}+\left(y_{j}-y_{k}\right)^{2} .
\end{aligned}
$$

Let

$$
H_{\mathrm{p}}=-\frac{1}{4 \pi} \sum_{j=1}^{N} \sum_{k>j} \Gamma_{k} \Gamma_{j} \log R_{j k}^{2}
$$

and

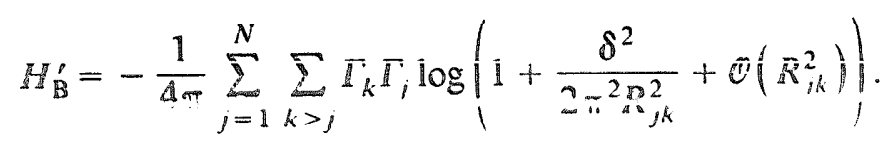

We have the following lemma:

Lemma 1. If $\delta \ll R_{j k} \ll 1$, then $H_{\mathrm{B}}$ is a small perturbation of the $N$-point Hamiltonian, $H_{\mathrm{p}}$ given by (4.6) and (2.1). 
In terms of the canonical coordinates (2.2) and the analysis in section 3, we can write the Hamiltonian $H_{\mathrm{B}}$ as follows:

$$
\begin{aligned}
& H_{\mathrm{B}}=H_{0}(\rho)+H_{1}(\rho), \\
& H_{0}(\rho)=-\frac{1}{4 \pi} \sum_{k=1}^{N-1} T_{k} \log \left|\rho_{k}\right|^{2}, \\
& T_{k}=\Gamma_{k+1} \sum_{k=1}^{k} \Gamma_{j}, \\
& H_{1}(\rho)=-\frac{1}{4 \pi} \sum_{k=1}^{M} T_{k} \log |(1+\mathcal{O}(\Delta))|^{2}+H_{\mathrm{B}}^{\prime},
\end{aligned}
$$

where $H_{1}(\rho)$ is the sum of two small terms (3.4d) and (4.7). The distances $R_{j k}$ in $H_{\mathrm{B}}^{\prime}$ can be expressed in the JL-coordinates in terms of the functions defined in (3.1) and (3.2), i.e.

$$
R_{j k}^{2}=f_{i}\left(\rho_{1}, \ldots, \rho_{i}\right) \overline{f_{i}\left(\rho_{1}, \ldots, \rho_{i}\right)},
$$

where each pair $(j, k)$ is associated with an integer $i \leq M=N(N-1) / 2$. Combining lemma 1 and the analysis in section 3 for $H_{\mathrm{p}}$, we obtain:

Lemma 2. In the cone sets

$$
M_{\Delta}^{\varepsilon}=\left\{\rho=\left(\rho_{1}, \ldots, \rho_{N-1}\right) \in \mathbb{C}^{N-1}|0<| \rho_{1}|<\Delta| \rho_{2}\left|<\cdots<\Delta^{N-2}\right| \rho_{N-1} \mid<\Delta^{N-2} \varepsilon\right\}
$$

for sufficiently small $\Delta$ and $\varepsilon$, the vortex blob Hamiltonian, $H_{\mathrm{B}}(\delta)$ is a small perturbation of a completely integrable term given by $(4.8 \mathrm{~b})$ provided the desingularization parameter $\delta$ is sufficiently small compared with $\varepsilon$.

Proof. The additionai condition on the absolute size of the coordinates $\left|\rho_{k}\right|$ given by $\Delta \sim \varepsilon \ll 1$ ensures that $\mathbb{R}_{j k} \sim \varepsilon$ satisfy the hypothesis in Lemma 1 .

Remark. The cone set (4.10) differs slightly from the cone set (3.3). In particular (4.10) is a proper subset of (3.3). Thus the analysis in section 3 can be used here. The additional condition in (4.10), $R_{j k} \ll 1$, imposes an admissible geometrical restriction on the vortex sheet discretization. It amounts to a non-uniform mesh as described in the numerical experiment proposed below.

Remark. The vortex blob model (4.3) does not require the vortex blobs to be widely separated either in the initial data or the subsequent evolution. Thus there is no contradiction between the restriction mentioned in the previous remark and the requirements of the model. On the other hand, the DEMM model requires the vortex patches to be widely separated, but in that case we fortunately do not have the above restriction.

The main result on the vortex blob model is:

Theorem 3. If the circulations $\Gamma_{j}$ satisfy

$$
\mathbb{T}_{k}=\Gamma_{k+1} \sum_{j=1}^{k} \Gamma_{j} \neq 0, \quad k=1, \ldots, N-1,
$$


then the Hamiltonian $H_{\mathrm{B}}(\delta),(4.3)$, supports a positive measure set of quasi-periodic solutions in the cone set $M_{\Delta}^{\varepsilon}$, (4.10), for sufficiently small $\delta \ll \varepsilon \ll 1$.

Proof. In the cone set, Hamiltonian $H_{\mathrm{B}}(\delta)$ is real analytic in the actions $\left|\rho_{k}\right|$ and $2 \pi$-periodic in the conjugate angle variables given by $\rho_{k}=\left|\rho_{k}\right| \mathrm{e}^{\mathrm{i} \theta_{k}}$. The leading term $H_{0}(\rho)$ has solutions $T^{N-1}$ parametrized continuously by $\left\{\left|\rho_{k}\right|\right\}_{k=1}^{N-1}$. It remains to verify that $H_{0}$ satisfies the KAM nondegeneracy condition, i.e. nonvanishing Hessian $\left|\partial^{2} H_{0} / \partial\right| \rho_{j}|\partial| \rho_{k}||$ when conditions (4.11) hold, which is an easy calculation.

Remark. By theorem 3, the Hamiltonian (4.3) supports quasi-periodic solutions if condition (4.11) is satisfied. At this point it is unclear whether the quasi-periodic solutions in the cone set (4.10) are related to the double spirals observed in the vortex sheet roll-up [2].

Remark. These quasi-periodic solutions lie on KAM tori within the cone set. This means that the cone condition (4.10) is preserved by the dynamics of (4.3) for a positive measure subset of all initial data satisfying the condition.

The above analysis suggests the following numerical experiment for a periodic vortex sheet:

On a non-uniform $\Gamma$-mesh where the discretization points are separated by a monotonically increasing sequence of intervals consistent with the ordering prescribed by the cone condition (4.10), carry out numerical computations for the desingularized vortex sheet (4.1). The circulations $\Gamma_{k}$ for this non-uniform mesh will be ordered to compensate for the clustering of the mesh points. For initial data on a slightly perturbed periodic vortex sheet, it shall be interesting to observe whether roll-up occurs in a manner consistent with the quasi-periodic behaviour predicted by theorem 3 .

5. The desingularized elliptic moment model

The Hamiltonian for the DEMM [11] is given by

$$
\begin{aligned}
H_{\mathrm{D}}= & H_{\mathrm{S}}+H_{\mathrm{P}}+H_{\mathrm{C}}, \\
H_{\mathrm{S}}= & -\frac{1}{8 \pi} \sum_{k=1}^{N} \Gamma_{k}^{2} \log \left(1+\frac{2 \pi}{A_{k}}\left(\delta_{k}^{2}+\gamma_{k}^{2}\right)\right), \\
H_{\mathrm{p}}= & -\frac{1}{8 \pi} \sum_{j, k=1}^{N} \Gamma_{j} \Gamma_{k} \log R_{j k}^{2}, \\
H_{\mathrm{C}}= & \frac{1}{\delta \pi} \sum_{j, k=1}^{N} \frac{\Gamma_{j} \Gamma_{k}}{R_{j k}^{2}}\left[\sqrt{\frac{A_{j}}{2 \pi}\left(1+\frac{2 \pi\left(\delta_{j}^{2}+\gamma_{j}^{2}\right)}{A_{j}}\right)}\left(\delta_{j} \cos 2 \theta_{k j}+\gamma_{j} \sin 2 \Theta_{k j}\right)\right. \\
& \left.+\sqrt{\frac{A_{k}}{2 \pi}\left(1+\frac{2 \pi\left(\delta_{k}^{2}+\gamma_{k}^{2}\right)}{A_{k}}\right)}\left(\delta_{k} \cos 2 \theta_{k j}+\gamma_{k} \sin 2 \theta_{k j}\right)\right],
\end{aligned}
$$




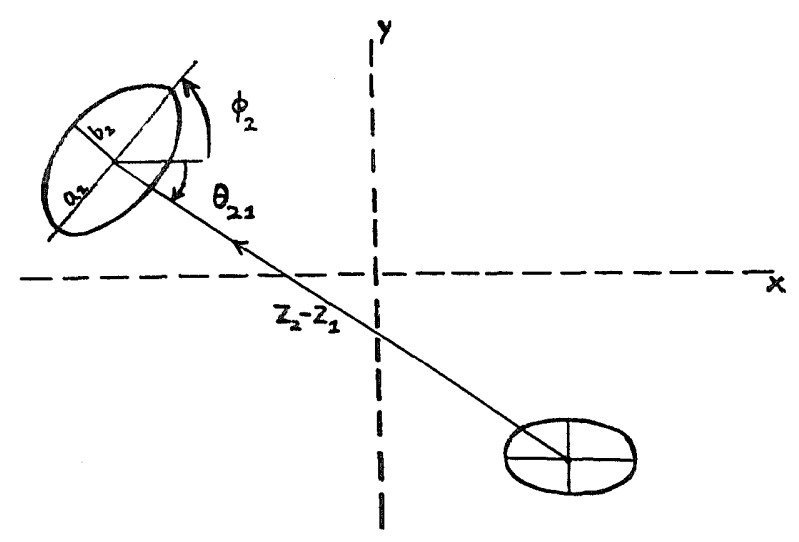

Fig. 2. Parameters for DEMM. The internal and external degrees of freedom for the DEMM are shown in the case of two vortex patches. $Z_{i}, i=1,2$, denotes the locations of the centroids and the aspect ratios are given by $a_{i} / b_{i}, i=1,2$.

where the conjugate variables are

$$
\left(\delta_{k}, \gamma_{k}\right)=\sqrt{\frac{A_{k}}{8 \pi \lambda_{k}}}\left(\lambda_{k}-1\right)\left(\cos 2 \phi_{k}, \sin 2 \phi_{k}\right)
$$

The following notation is used (see fig. 2):

$A_{k}$ is the area of the $k$ th vortex patch,

$\phi_{k}$ is the orientation of the patch,

$\lambda_{k}$ is the aspect ratio of the patch,

$R_{j k}$ is the inter-centroid distance and

$\theta_{j k}$ is the centroid-centroid angle between the $j$ th and $k$ th patches.

A basic assumption in the derivation of $(5.1)$ is that [11]

$$
\eta=\frac{A_{k}}{R_{j k}^{2}} \ll 1 \text { for all } j \neq k,
$$

which states that the vortex patches are widely separated compared to their sizes.

The DEMM Hamilionian $H_{\mathrm{D}}$ has $N$ external degrees of freedom

$$
z_{k}=x_{k}+\mathrm{i} y_{k}, \quad k=1, \ldots, N
$$

and $N$ internal degrees of freedom

$$
v_{k}=\delta_{k}+\mathrm{i} \gamma_{k}, \quad k=1, \ldots, N
$$


We are ready to put $H_{\mathrm{D}}$ in the complex formulation (from which the original Hamiltonian is recovered by taking the real part):

$$
\begin{aligned}
& H_{\mathrm{D}}=H_{\mathrm{S}}+H_{\mathrm{P}}+H_{\mathrm{C}} \\
& H_{\mathrm{S}}=-\frac{1}{8 \pi} \sum_{k=1}^{N} \Gamma_{k}^{2} \log \left(1+2 \pi \frac{v_{k} \bar{v}_{k}}{A_{k}}\right) \\
& H_{\mathrm{P}}=-\frac{1}{8 \pi}: \sum_{j, k=1}^{N} \Gamma_{j} \Gamma_{k} \log \left(z_{j}-z_{k}\right) \\
& H_{\mathrm{C}}=\frac{1}{4 \pi} \sum_{j, k=1}^{N} \frac{\Gamma_{j} \Gamma_{k}}{\left(z_{j}-z_{k}\right)^{2}}\left[\sqrt{A_{k}}\left(1+\frac{2 \pi}{A_{k}} v_{k} \bar{v}_{k}\right)^{1 / 2} v_{k}+\sqrt{A_{j}}\left(1+\frac{2 \pi}{A_{j}} v_{j} \bar{v}_{j}\right)^{1 / 2} v_{j}\right]
\end{aligned}
$$

$H_{\mathrm{S}}$ is the self-interaction term which is cyclic in the angle variables $2 \phi_{k}(5.2) . H_{\mathrm{P}}$ is the usual $N$-point vortex Hamiltonian and $H_{\mathrm{C}}$ is the term that couples the external and internal degrees of freedom.

The second step is a transformation given by

$$
\left\{\sqrt{\lambda_{k}} z_{k}\right\}_{1}^{N} \rightarrow\left\{\rho_{k}\right\}_{1}^{N}, \quad\left\{v_{k}\right\}_{k=1}^{N} \rightarrow\left\{v_{k}\right\}_{k=1}^{N} \text { unchanged }
$$

where the external variables are transformed to the JL-coordinates (2.2) and the internal variables are unchanged.

Lemma 4. The real and imaginary parts of $\left\{\rho_{k}\right\}_{k=1}^{N}$ and $\left\{v_{k}\right\}_{k=1}^{N}$ are canonically conjugate. The transformed Hamiltonian in $(2 N-1)$ degrees of freedom is

$$
H_{\mathrm{D}}(\rho ; v)=H_{\mathrm{S}}(v)+H_{\mathrm{P}}(\rho)+H_{\mathrm{C}}(\boldsymbol{p} ; \boldsymbol{v})
$$

where $H_{\mathrm{S}}(v)$ is given by $(5.4 \mathrm{~b}), H_{\mathrm{P}}(\rho)$ is given by $(3.1)$ and

$$
H_{\mathrm{C}}(\rho ; v)=\frac{1}{4 \pi} \sum_{j, k=1}^{N} \frac{\Gamma_{j} \Gamma_{k}}{\left(f_{i}(\rho)\right)^{2}}\left[\sqrt{A_{k}}\left(1+\frac{i \pi}{A_{k}} v_{k} \bar{v}_{k}\right)^{1 / 2} v_{k}+\sqrt{A_{j}}\left(1+\frac{2 \pi}{A_{j}} v_{j} \bar{v}_{j}\right)^{1 / 2} v_{j}\right]
$$

Sketch of proof. Since the self-interaction term $H_{\mathrm{S}}$ involves only internal degrees of freedom $\left\{v_{k}\right\}_{k=1}^{V}$ which are unchanged under (5.5), this term is transformed without change. Next the point-vortex term $H_{p}(\mathbb{2})$ depends only on the external degrees of freedom and it has been shown to transform into (3.1) [9]. It remains to verify that the coupled parts of the vector field can be written in Hamiltonian form in terms of the variables (5.5). This is where the complex formulation for the coupling term $H_{C}(z ; v),(5.4 d)$, facilitates the otherwise tedious but straightforwati calculations. Note that in terms of $\left\{z_{k}\right\}$ and $\left\{v_{k}\right\}, H_{C}$ is a sum of quotients, the numerator involving only the internal degrees of freedom and the denominator has the simple quadratic form $\left(z_{j}-z_{k}\right)^{2}=f_{i}^{2}\left(\rho_{1}, \ldots, \rho_{k}\right)$ where $f_{i}$ are linear in the JL-coordinates. The lengthy details are omitted. 
In the third step, we construct special open sets in phase space that are products of cone sets (3.3) for the external variables and a polydisk for the internal variables,

$$
\begin{aligned}
& M_{\varepsilon}^{\Delta}=M_{\Delta} \times P_{\varepsilon}, \\
& P_{\varepsilon}=\left\{\left.\left(v_{1}, \ldots, v_{N}\right) \in \mathbb{C}^{N}|| v_{k}\right|^{2} / A_{k} \leq \varepsilon\right\} .
\end{aligned}
$$

Remark. The cone set (3.3) in the product (5.7) does not have the additional restriction of (4.10). Thus, it is compatible with the DEMM requirement that the vortex patches are widely separated (5.3).

The aim here is to write $H_{\mathrm{D}}$ as a small perturbation of a decoupled term $H_{0}^{\prime}(\rho)$ plus the self-interaction term $H_{\mathrm{s}}\left(\left|v_{k}\right|^{2}\right)$.

Lemma 5. If the basic assumption in DEMM, $A_{k} / R_{j k}^{2}=\eta \ll 1(5.3)$, holds for all $j \neq k$, then for sufficiently small $\Delta$ and $\varepsilon$, the Hamiltonian $H_{\mathrm{D}}(\rho ; v),(5.6 \mathrm{a})$, can be written as a small perturbation of a completely integrable term in the open set $M_{\varepsilon}^{\Delta}$ defined in (5.7):

$$
\begin{aligned}
& H_{\mathrm{D}}=H_{0}\left(\left|\rho_{k}\right|^{2} ;\left|v_{k}\right|^{2}\right)+H_{1}(\rho ; v), \\
& H_{0}=H_{0}^{\prime}\left(\left|\rho_{k}\right|^{2}\right)+H_{\mathrm{S}}\left(\left|v_{k}\right|^{2}\right), \\
& H_{0}^{\prime}=-\frac{1}{4 \pi} \sum_{k=1}^{N-1} T_{j} \log \left(\left|\rho_{k}\right|\right)^{2}, \\
& H_{1}=H_{1}^{\prime}(\rho)+H_{\mathrm{C}}(\rho ; v), \\
& H_{1}^{\prime}(\rho)=-\frac{1}{4 \pi} \sum_{j=1}^{M} \mathbb{K}_{j} \log |(1+\mathcal{O}(\Delta))|^{2},
\end{aligned}
$$

where $H_{\mathrm{S}}$ is given by $(5.1 \mathrm{~b}), H_{\mathrm{C}}$ is given by (5.1d) and $H_{0}, H_{1}$, are given by (3.4).

Proof. Under the assumption (5.3) and for sufficiently small $\varepsilon$ in (5.7) the nonlinear coupling $H_{\mathrm{C}}$ is of order $(\varepsilon \eta)$ by inspection. In the cone set $M_{\Delta}$ for $\Delta$ sufficiently small $H_{1}^{\prime}$ is of order $\Delta$. Therefore the perturbation $H_{1}$ in (5.8a) is small under the above assumptions. The leading term $H_{0}$ is clearly completely integrable because $H_{0}^{\prime}$ is decoupled and $H_{S}$ depends only on the actions $\tilde{I}_{k}=\delta_{k}^{2}+\gamma_{k}^{2}$.

The main result for the DEMM follows from KAM-theory.

Theorem 6. If $\varepsilon, \Delta$ and $\eta$ are all sufficiently small, then the DEMM Hamiltonian (5.1) supports a positive measure set of quasi-periodic solutions in the open set $\mathrm{M}_{\varepsilon}^{A},(5.7)$, provided the following conditions hold:

$$
\mathbb{T}_{k}=\Gamma_{k+1} \sum_{j=1}^{k} \Gamma_{j} \neq 0 \quad \text { for } k=1, \ldots, N-1
$$

Proof. In the cone set $M_{e}^{\Delta}$, the DEMM Hamiltonian, $H_{\mathrm{D}}$ is real analytic in the actions, $\left|\rho_{k}\right|^{2}, \mathbb{H}_{k}=\delta_{k}^{2}+\gamma_{k}^{2}$ and $2 \pi$-periodic in the conjugate angles $\theta_{k}$ and $\phi_{k}$ where $\rho_{k}=\left|\rho_{k}\right| \mathrm{e}^{\mathrm{i} \theta_{k}}$ and $\phi_{k}$ are defined in (5.2). The 
unperturbed term $H_{0},(5.8 \mathrm{~b})$, has solutions

$$
T^{N-1}\left(\left\{\theta_{k}\right\}\right) \times T^{N}\left(\left\{\phi_{k}\right\}\right)
$$

parametrized continuously by $\left\{\left|\rho_{k}\right|^{2}\right\}_{k=1}^{N-1}$ and $\left\{I_{k}\right\}_{k=1}^{N}$. The KAM-nondegeneracy condition is easily verified because $H_{0}(5.8 \mathrm{~b})$ is decoupled in all the actions, i.e. the Hessian is diagonal. $\partial^{2} H_{0} / \partial\left(\left|\rho_{k}\right|\right)^{2} \neq 0$ when (5.9) holds and $\partial^{2} H_{0} / \partial I_{k}^{2} \neq 0$ provided $\Gamma_{k}^{2} \neq 0$ which is implied by (5.9).

An important corollary of this result is the rigorous validification of the DEMM in the following sense:

Corollary. The basic assumption (5.3) in the derivation of the DEMM is consistent for a positive measure set of solutions, i.e. quasi-periodic solutions on the KAM-tori satisfy (5.3) for all time.

\section{Acknowledgements}

The author would like to thank Robert Krasny for helpful discussions on the vortex sheet problem and Christoph Borgers for his preprint. He would also like to thank Dee Hudock for the professional typing of this manuscript.

\section{References}

[1] R. Krasny, J. Fluid Mech. 167 (1986) 65.

[2] R. Krasny, J. Comp. Phys. 65 (1986) 292.

[3] R. Caflisch and J. Lowengrub, Convergence of the vortex method for vortex sheets, preprint.

[4] D.I. Pullin, J. Fluid Mech. 119 (1982) 507.

[5] P.G. Saffman and G.R. Baker, Ann. Rev. Fluid Mech. 11 (1979) 95.

[6] G. Joyce and D. Montgomery, J. Plasma Phys. 10 (1973) 107.

[7] Y.B. Pointin and T.S. Lundgren, Phys. Fluids 19 (1976) 1459.

[8] Ch.C. Lim, Singular manifolds and quasi-periodic solutions of Hamiltonians for vortex lattices, Physica D 30 (1988) 343.

[9] Ch.C. Lim, Existence of KAM tori in the phase-space of vortex systems, preprint.

[10] Ch.C. Lim and L. Sirovich, Nonlinear vortex trail dynamics, Phys. Fluids (May 1988).

[11] M.V. Melander, N.J. Zabusky and A.S. Styczek, J. Fluid Mech. 167 (1986) 95.

[12] Ch.C. Lim, On singular Hamiltonians: the existence of quasi-periodic solutions and nonlinear stability, Bull. AMS 20 (1989) 35.

[13] V.I. Arnol'd, Russ. Math. Survey 18(5) (1963) 9.

[14] J. Moser, Stable and Random Motions in Dynamical Systems (Princeton Univ. Press, Princeton, NJ, 1973).

[15] G. Birkhof, Helmholtz and Taylor Instability, in Proc. of the Symp. on Appl. Math., vol. XIII. (A.M.S., Providence, RI, 1962).

[16] C. Borgers, On the numerical solution of the regularized Birkhoff equations, preprint.

[17] Ch.C. Lim, Canonical transformations and graph theory, to appear in Phys. Lett. A. 